\title{
Frequency control in subterahertz gyrotrons
}

\author{
A. Bogdashov, A. Fokin, A. Fedotov, M. Glyavin, M. Morozkin, Yu. Novozhilova, \\ M. Proyavin, R. Rozental, A. Sedov, A. Tsvetkov, I. Zotova and G. Denisov
}

Institute of Applied Physics of the Russian Academy of Sciences, Nizhny Novgorod, Russia, ap.fokin@ mail.ru

Subterahertz gyrotrons are promising for application in different fields of science and technology, like spectroscopy [1], media diagnostics [2] and materials processing [3]. Many of the application require the possibility of precise frequency tuning or stabilization. In this paper we present the experimental investigation of different ways of frequency control in medium-power continuous-wave gyrotrons carried out in the Institute of Applied Physics RAS. The capabilities and limitations of different methods are presented.

\section{Magnetic field variation}

The magnetic field variation provides the biggest range of frequency tuning. Excitation of different transverse modes with close caustic radius enables step frequency tuning in range up to $200 \mathrm{GHz}$ [4]. The fine frequency tuning with band of up to $6 \%$ of carrier frequency can be obtained by consequent excitation of high-order longitudinal modes. It is possible due to the use of a short cavity with low Qfactor and operation at low transverse modes in order to increase the electron-wave coupling. The proof of concept experiment on low frequency (using the $12 \mathrm{GHz}$ gyrotron setup) demonstrated the $4 \%$ tuning band without any special optimization of the system (see Fig. 1). Based on this concept the project of the $200 \mathrm{GHz}$ gyrotron with $1 \mathrm{~kW}$ output power and 10 $\mathrm{GHz}$ band was developed [5].

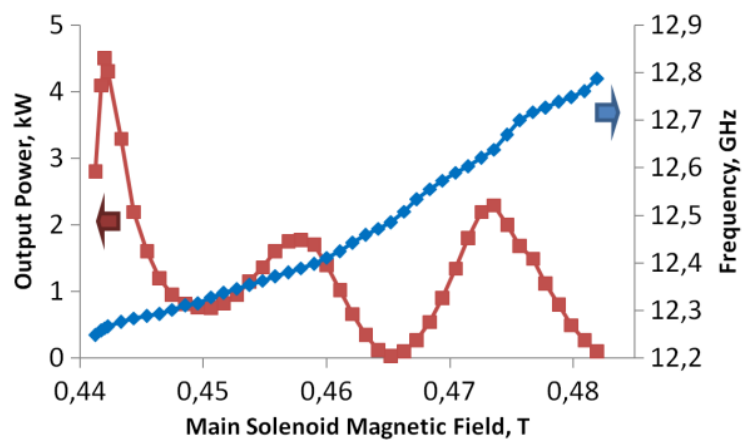

Fig. 1 The gyrotron output power and frequency vs. magnetic field

The frequency tuning speed of such method is limited by the parameters of the cryomagnets, mainly the maximum current variation rates, which are less than $0.1 \%$ of maximum field per minute. The possible solution is to apply the additional coil either in the cathode region or at the cavity. The latter approach was tested and demonstrated the possibility of operating mode switching with sweeping frequency up to $2 \mathrm{kHz}$. The same method can be applied for fast power modulation with frequency up to $10 \mathrm{kHz}$ (Fig. 2), which is limited by auxiliary coil inductance and alternating filed screening [6].

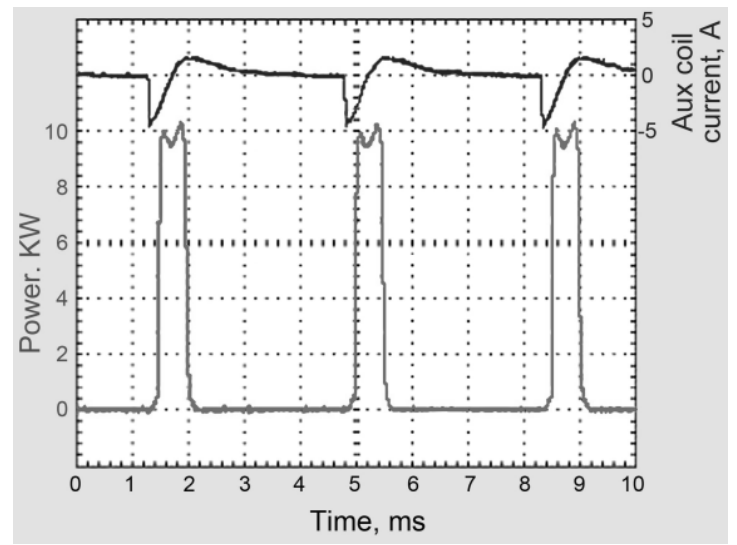

Fig. 2 Oscillograms of auxiliary coil current (top) and gyrotron output power (bottom)

\section{Cavity temperature variation}

In the recent experiments, the possibility of tuning the frequency of the $0.26 \mathrm{THz}$ gyrotron [7] by more than $1 \mathrm{GHz}$ was demonstrated by the simultaneous changing of the magnetic field of the main solenoid and the temperature of the cavity coolant. The measured frequency sensitivity of $4 \mathrm{MHz} /{ }^{\circ} \mathrm{C}$ allowed to tune the frequency in more than $200 \mathrm{MHz}$ range and close the power gap between different longitudinal modes (Fig. 3).

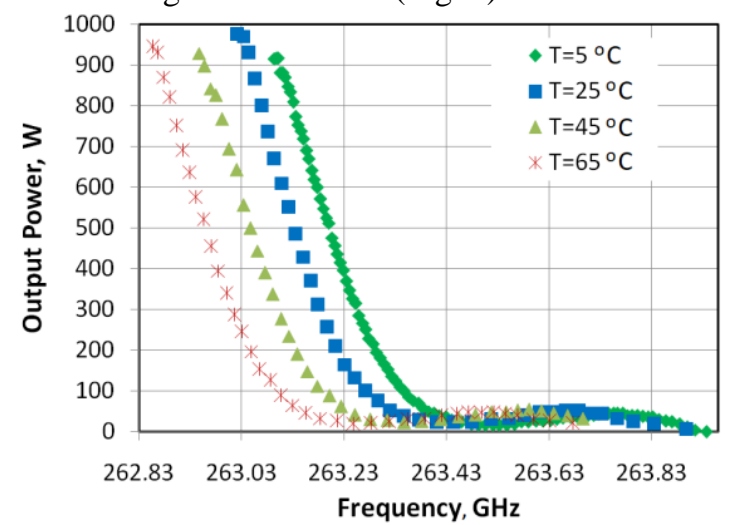

Fig. 3. Output power vs. frequency for different cavity coolant temperatures

\section{Voltage variation}

The fastest way of frequency control is the variation of the voltage at one of the electrodes of the magnetron-injection gun or the potential of the gyrotron cavity [8, 9]. The modulation of cathode voltage requires complex and expensive power supply; the speed of cavity voltage variation is limited 
by big capacitance, so the most effective way of frequency control is the anode voltage variation.

For this purpose, the special control unit was developed, that allowed voltage variation in range of $1 \mathrm{kV}$ with speed more than $1 \mathrm{kV} / \mu \mathrm{s}$. The control system based on this unit opened up the possibility of frequency tuning in 20 to $50 \mathrm{MHz}$ range with up to $200 \mathrm{kHz}$ modulation frequency. Application of the system for frequency stabilization by phase-lock loop against reference oscillator allowed to achieve the spectrum width of the gyrotron radiation of $1 \mathrm{~Hz}$ [10].

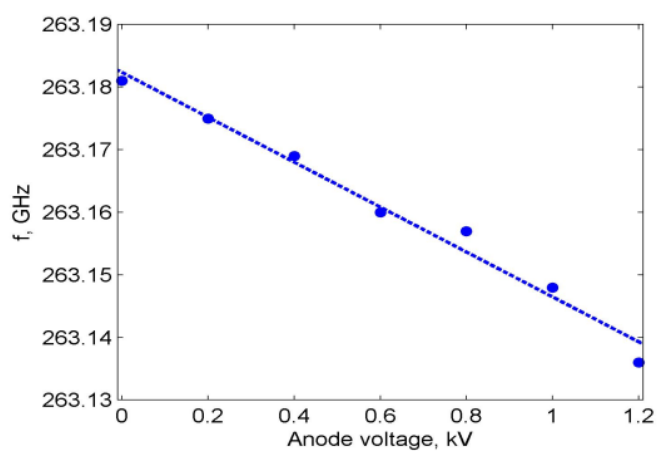

Fig. 4. Radiation frequency of a gyrotron vs. anode voltage

\section{External signal and reflections}

The presence of external signal or the power reflected from the load can be used for power and frequency control [11]. Experiments on influence of the signal, reflected from distant non-resonant load [12] show the possibility of frequency stabilization, while proof of concept experiments with mobile reflector demonstrate the means of slow power and frequency control (Fig. 5)

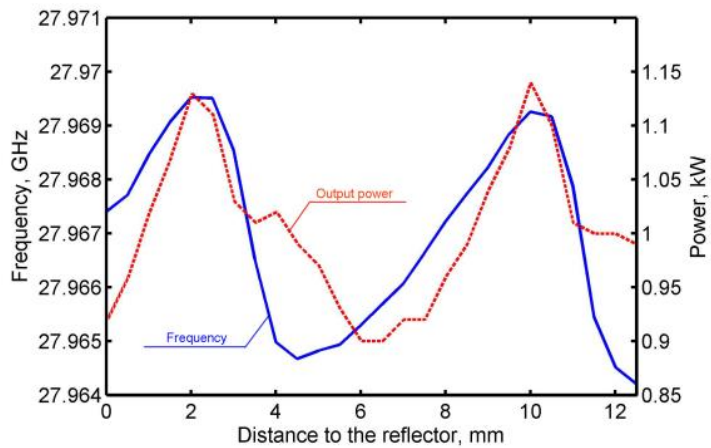

Fig. 5. Output power and frequency vs. distance to the nonresonant reflector

\section{Conclusion}

The IAP RAS gyrotron research team developed and tested a number of approaches to frequency control in sub- $\mathrm{THz}$ gyrotrons, including excitation of high-order longitudinal modes, cavity temperature sweeping, anode voltage variation and use of reflections. Beyond the scope of this work are mechanical means of frequency tuning, for example [13], which are surely promising, but their use in sub-THz frequency range is limited by the precision requirements.

Investigated approaches open up new prospects for the successful development of new medium power $\mathrm{THz}$ band gyrotrons with unique capabilities for modern applications

The work was supported by Russian Foundation for Basic Research grant № 17-02-00183

\section{References}

1. Blank M, Rosay $M$, Engelke $F$ Instrumentation for solid-state dynamic nuclear polarization with magic angle spinning NMR // J. Magn. Reson., 2016, V. 264 P. 88-98

2. Yamaguchi, Y. et al., High-power pulsed gyrotron for $300 \mathrm{GHz}$-band collective Thomson scattering diagnostics in the Large Helical Device // Nuclear Fusion, 2015, V. 55 013002

3. Vodopyanov, A. et al., Application of the $263 \mathrm{GHz} / 1$ $\mathrm{kW}$ gyrotron setup to produce a metal oxide nanopowder by the evaporation-condensation technique // Vacuum, 2017, V. 145 , P. $340-346$

4. Idehara, T. et al., A novel THz-band double-beam gyrotron for high-field DNP-NMR spectroscopy // Review of Scientific Instruments, 2017, V. 88, 094708

5. Fedotov, A.E. et al., Frequency Tunable sub-THz Gyrotron for Direct Measurements of Positronium Hyperfine Structure // Journal of infrared, millimeter and terahertz waves, 2018, V. 39, No. 10, P. 975-983

6. Glyavin, M.Yu., Luchinin, A.G., Morozkin, M.V., Ka-band $10 \mathrm{~kW} \mathrm{CW}$ gyrotron with the wide-band fast frequency sweep // Rev. Sci. Instrum., 2012, V. 83, 074706

7. Glyavin M.Y. et al. Experimental tests of $263 \mathrm{GHz}$ gyrotron for spectroscopy applications and diagnostic of various media // Review of Scientific Instruments, 2015, V. 86, No. 5, P. 054705

8. Idehara, T., Mitsudo, S., Ogawa, I. Development of High-Frequency, Highly Stable Gyrotrons as Millimeter to Submillimeter Wave Radiation Sources // IEEE Trans. Plasma Sci., 2004, V. 32, P. 910-916

9. Golubiatnikov, G.Y. et al. Gyrotron frequency control by a phase lock system // Tech. Phys. Lett., 2006, V. 32, P. 650

10. Fokin, A. et al., High-power sub-terahertz source with a record frequency stability at up to $1 \mathrm{~Hz} / /$ Scientific Reports, 2018, V. 8, 4317

11. Novozhilova, Yu. et al., Gyrotron frequency stabilization under the influence of external monochromatic signal or wave reflected from the load: Review // Izvestiya VUZ, Applied Nonlinear Dynamics, 2017, V. 25, No. 1, P. 4-34

12. Glyavin, M.Yu. et al., Frequency Stabilization in a Sub-Terahertz Gyrotron With Delayed Reflections of Output Radiation // IEEE Transactions on Plasma Science, 2018, V. 46, No. 7, P. 2465 - 2469

13. Sabchevski, S, Idehara, T. Resonant Cavities for Frequency Tunable Gyrotrons // Int J Infrared Milli Waves, 2008, V. 29, P. 1-22 